1 Universidade Federal da Bahia (UFBA), Instituto de Saúde Coletiva (ISC) Salvador (BA), Brasil. catharinamatos@gmail.com

2 Fundação Oswaldo Cruz (Fiocruz), Coordenação de Educação da Vice-

Presidência de Educação e Comunicação - Rio de Janeiro (RJ), Brasil.

\section{Implantação da acreditação pedagógica de cursos lato sensu em Saúde Pública no Brasil: desafios e perspectivas}

\author{
Implementation of pedagogical accreditation of lato sensu courses in \\ Public Health in Brazil: challenges and perspectives
}

Catharina Matos', Isabela Cardoso de Matos Pinto', Tania Celeste de Matos Nunes ${ }^{\mathbf{2}}$

DOI: 10.1590/0103-11042019S103

RESUMO O objetivo do artigo foi analisar a implantação da acreditação pedagógica no Brasil, no período de 2014 a 2018. Realizou-se análise documental para produção dos dados, a partir dos relatórios elaborados no período, buscando identificar os principais problemas referentes a essa implantação na dimensão da gestão mais ampla e quanto aos processos de acreditação. Os resultados apontaram problemas de várias ordens e efeitos positivos para o processo, para os atores institucionais e para o Sistema Único de Saúde (SUS). Os resultados mostram que há problemas de ordem gerencial, pedagógica e de infraestrutura, entretanto as acreditações produziram efeitos positivos para as escolas, para os trabalhadores e para o SUS.

PALAVRAS-CHAVE Acreditação pedagógica. Lato sensu. Sistema Único de Saúde.

ABSTRACT The purpose of the article was to analyze the implementation of Pedagogical Accreditation in Brazil, from 2014 to 2018. A documentary analysis was carried out to produce the data, based on the reports elaborated in the period, seeking to identify the main problems related to this implementation in both the broader management dimension, as well as regarding the accreditation processes. The results pointed to problems of several orders and positive effects for the process, for the institutional actors and for the Unified Health System (SUS). The results show that there are management, pedagogical and infrastructure problems. However, accreditation has produced positive effects for schools, for workers and for the SUS.

KEYWORDS Pedagogical accreditation. Lato sensu. Unified Health System. 


\section{Introdução}

A gestão do trabalho e da educação na saúde é um dos desafios enfrentados no processo de construção do Sistema Único de Saúde (SUS), demandando continuada reflexão sobre a articulação entre a reorganização das práticas de atenção à saúde e as mudanças e transformações necessárias nos processos de formação de pessoal.

Nesses 30 anos do SUS, várias iniciativas têm sido desenvolvidas em todo o País em torno do papel das instituições gestoras da saúde na formulação e implementação de políticas de formação de pessoal, constituição de redes descentralizadas de formação das equipes multiprofissionais e dos trabalhadores de saúde, conformação de espaços de integração ensino-serviço, reorientação dos processos de educação permanente dos trabalhadores da saúde, implantação de novos cursos de graduação e de pós-graduação, reorientação dos currículos dos cursos existentes, e a incorporação de novas tecnologias no processo educacional, entre outras ${ }^{\mathbf{1}, \mathbf{2}}$.

A expansão dos cursos de especialização na área da saúde no Brasil, na segunda metade do século XX, e a preocupação com a articulação entre conteúdos curriculares e as práticas de saúde desencadearam o debate sobre a qualidade dos processos formativos, incluindo a implementação da Política Nacional de Educação Permanente em Saúde (PNEPS). Nesse sentido, a acreditação pedagógica consiste em uma estratégia que, entre outros objetivos, contribui para a avaliação das ações e práticas de Educação Permanente em Saúde (EPS). Essa interpretação parte do princípio que as avaliações em EPS estimulam os atores envolvidos nos processos educativos, assim como as instituições ofertantes para seu autoconhecimento e o reconhecimento das suas potencialidades e fragilidades, ampliando o compromisso social da instituição com a qualidade da implementação das políticas públicas afetas por seu objeto ${ }^{3}$.

A revisão de literatura sobre o tema da qualidade em processos educativos indica a expansão do movimento da acreditação pedagógica como estratégia de avaliação da qualidade, bem como evidencia que a sua incorporação tem influenciado a melhoria da qualidade nos serviços de saúde ${ }^{4-6}$.

Há evidências consistentes de que os programas de acreditação aprimoram o processo de atendimento prestado pelos serviços de saúde ${ }^{4-6}$. Tais evidência foram apontadas por Braithwaite e Greenfield ${ }^{6}$ em pesquisa realizada sobre acreditação em saúde, em que foram selecionados 66 artigos. Os autores concluíram que os programas de acreditação foram relacionados com melhor desempenho dos profissionais, uma vez que forneceram oportunidade de reflexão sobre suas práticas e sobre as mudanças organizacionais na implementação de programas contínuos de melhoria da qualidade, instigadas pela acreditação. A análise das informações dos sites de 22 agências afiliadas ao International Society for Quality in Health Care (ISQua) ainda corrobora essas evidências, cuja análise das informações atesta que os processos de acreditação promovem a mudança e o desenvolvimento profissional em escolas de ensino superior e serviços de saúde na Europa.

No Brasil, o processo de implantação da acreditação pedagógica dos cursos lato sensu em Saúde Pública e/ou Saúde Coletiva foi iniciado na década de 1990 na Escola Nacional de Saúde Pública Sergio Arouca da Fundação Oswaldo Cruz (Ensp-Fiocruz), mas foi interrompido por falta de condições objetivas para prosseguir em sua meta de institucionalização. Em 2009, esse tema voltou a ser debatido no âmbito do GT Trabalho e Educação da Associação Brasileira de Saúde Coletiva (Abrasco) e no interior da Rede de Escolas e Centros Formadores em Saúde Pública (RedEscola), com a criação de um Grupo de Trabalho (GT) da Acreditação Pedagógica sob a coordenação da Secretaria Técnica e Executiva Rede de Brasileira de Escolas de Saúde Pública. Nesse período, foi retomada a parceria com a École de Hautes Etudes em 
Santé Publique de Rennes na França, interrompida na década de 1990. O GT de Acreditação Pedagógica da Rede contou com a participação de um grupo de Escolas filiadas, sob a coordenação da Secretaria Técnica da Rede, e foi um mecanismo fundamental para a construção da viabilidade político-institucional da Agência de Acreditação Pedagógica no âmbito da Abrasco. Em 2016, limites jurídicos e administrativos da Abrasco para a constituição de uma Agência de Acreditação nos moldes internacionais resultaram na conformação de um Comitê de Acreditação vinculado ao GT de Trabalho e Educação da Abrasco.

Ao longo de quatro anos, foram acreditados cinco cursos de instituições formadoras que compõem a RedEscola, configurando um movimento de adesão à proposta e de incorporação dos princípios e valores que integram o processo acreditador, criando as condições para a criação de um Sistema Nacional de Acreditação (SNA), com vistas à melhoria da qualidade dos cursos e por consequência, dos serviços prestados à população no SUS.

Destarte, o presente artigo toma como referência a análise da experiência pela Abrasco, propondo-se a analisar a implantação da acreditação pedagógica no Brasil, no período de 2014 a 2018, apontando seus principais problemas. Ademais, buscou analisar os efeitos da acreditação pedagógica para as escolas, para os trabalhadores e para o SUS.

\section{Metodologia}

Analisou-se a implantação da acreditação pedagógica no Brasil, no período de 2014 a 2018. Para a produção das evidências, tomaram-se como referência os documentos produzidos pelas reuniões realizadas no âmbito da estrutura de governança do Comitê de Acreditação Pedagógica da Abrasco (CAP/Abrasco), notadamente o Conselho de Acreditação e o Comitê de Experts; bem como os relatórios anuais de atividades, os relatórios das oficinas de mobilização e os relatórios produzidos pelos avaliadores externos, durante as visitas com vistas à acreditação.

Para a sistematização dos problemas, adotou-se o referencial de Carlus Matus ${ }^{6}$. Para esse autor, três conceitos são fundamentais: a) ator social, que são pessoas, grupamento humano ou instituição com capacidade de produzir fatos na situação; b) os problemas, como tudo aquilo que se encontra fora do padrão de normalidade pelo ator social; e c) situação, entendida como um conjunto de problemas identificados, descritos e analisados na perspectiva de um determinado ator social. A seleção e a identificação de problemas sociais sob diferentes perspectivas favorecem a solução e o enfrentamento $\mathbf{7 , 8}$.

$\mathrm{Na}$ análise, os problemas foram processados em matriz de Excel $^{\circledR}$ contendo uma dimensão geral da implantação, uma dimensão do processo de acreditação e uma outra, para análise dos efeitos produzidos pela avaliação para as escolas, para os trabalhadores e para o SUS.

\section{Resultados e discussão}

\section{Implantação da acreditação peda- gógica: da proposta de Agência de Acreditação a constituição do Comitê de Acreditação da Abrasco}

Em 2014, foi iniciada a implantação da acreditação pedagógica, visando implantar uma Agência de Acreditação Pedagógica como resultado da articulação entre a RedEscola, a Abrasco e o Ministério da Saúde (MS), com o apoio técnico e científico da École de Hautes Étude en Santé Publique de Rennes-França. Os cursos a serem acreditados eram integrantes de Escolas vinculados à RedEscola, a Abrasco foi escolhida como lócus onde seria abrigada a Agência e o MS participou com o apoio financeiro do projeto que resultou dessa articulação.

A decisão de abrigar a agência na Abrasco encontrou respaldo no argumento de isenção dessa entidade do ponto de vista ético-político 
e pela legitimidade dessa entidade nas Escolas e Centros Formadores em Saúde Pública no Brasil. Essa decisão permitiu proteger a nova Agência de conflitos de interesses, que não devem existir nos processos de avaliação.

A configuração inicial de uma 'Agência' nos moldes internacionais adotados inicialmente com forte influência das entidades congêneres europeias esbarrou em limites jurídicos e gerenciais, representados pelas exigências para a criação de uma entidade jurídica, inclusive nos aspectos relativos à sustentabilidade financeira. Nesse sentido, a Abrasco adotou o formato de uma 'incubadora do projeto', viabilizando a experiência da acreditação dos cursos. Em 2016, estabeleceu-se um Comitê de Acreditação Pedagógica articulado ao GT Trabalho e Educação da Abrasco, com funções de agência acreditadora.

Esse Comitê, permitiu a organização de uma estrutura de governança que envolveu três instâncias fundamentais: a) uma Coordenação Executiva, b) um Comitê de Experts para Acreditação e c) um Conselho de Acreditação. Cada instância foi desenhada com atribuições específicas: a coordenação executiva coordenou a implantação do processo; o Conselho da acreditação respondeu pela política mais geral, deliberando sobre os assuntos como plano de trabalho, estratégias de articulação, entre outros assuntos, sempre relativos às tomadas de decisões; e, por último, o Comitê de Experts que se responsabilizou por validar a acreditação, dando a chancela aos cursos em processo de acreditação, previamente analisados pelos avaliadores externos.

\section{Implantação da acreditação pedagó- gica: problemas encontrados}

A acreditação pedagógica se inicia com a adesão voluntária das escolas que constituem a RedEscola. O critério para o ingresso no SNA é a realização de um curso de especialização em caráter perene, pelo menos, três anos consecutivos. Essa medida se inspira no fato de os cursos de especialização na modalidade lato sensu caracterizarem-se por ofertas irregulares, em áreas distintas e, muitas vezes, definidas por editais ${ }^{3}$. No caso da acreditação pedagógica, as disparidades regionais entre as escolas que compõem a rede são grandes e se refletem nas suas respectivas ofertas educativas. Nesse caso, um problema vivenciado estava relacionado com o fato de que poucas instituições apresentavam cursos em condições de elegibilidade, muito embora algumas delas tivessem intenção em compor o SNA.

Uma vez aceita a solicitação, inicia-se o processo de acreditação por meio da produção de um relatório de autoavaliação. Esse momento representa o encontro dos atores institucionais para discussão dos problemas que envolvem o curso colocado em processo de acreditação. Embora seja o curso a unidade sob acreditação, o movimento da autoavaliação que se realiza em torno da produção do relatório corresponde a uma análise crítica de toda a instituição que oferta o curso. Em geral, há o envolvimento de atores - de gestão mais ampla, coordenação e operacionalização - que ocupam diferentes posições no curso.

O relatório de autoavaliação é o documento base para toda a acreditação. Esse documento é enviado pela Escola à Secretaria Executiva do Comitê de Acreditação Pedagógica, que, na sequência, o encaminha a uma comissão de avaliação externa, previamente escolhida pela Secretaria Executiva do Comitê de Acreditação Pedagógica. Essa comissão é constituída por atores que passam por qualificação específica oferecida pela RedEscola em articulação com o Comitê de Acreditação, habilitando-se para o desenvolvimento dessa tarefa. Na produção desse relatório, o principal problema disse respeito ao seu tempo de produção, que, em alguns casos, chegou a durar seis meses. Como consequência, houve alongamento do tempo de acreditação compreendido entre o momento da adesão até a decisão por acreditação, emitido pelo Comitê de Experts. Esses resultados contrapõem-se ao modelo internacional que adota prazos e medidas punitivas definidas. Resultados de 
avaliações realizadas por agências internacionais mostram que quando não há sanções, não há efeitos positivos para as instituições e para os processos educativos ${ }^{9}$.

O processo avança com a análise do relatório de autoavaliação e outros documentos enviados à comissão de avaliadores, previamente definida e designada pelo Comitê. Uma vez analisados os referidos documentos, ocorre a avaliação externa de modo presencial, em visita à instituição candidata, em geral por três dias. Essa equipe também emite relatório de avaliação externa, enviada ao Comitê de Experts, que chancela ou não o relatório produzido, e conclui pela acreditação ou não do curso em análise. Nesse aspecto, o principal problema foi o reduzido número de avaliadores externos, assim como a sua localização no âmbito das escolas que compõe a RedEscola, pois exigiram cuidados para não produzir conflitos de interesse.

Todo o processo foi ancorado em dois instrumentos básicos: o Referencial de Qualidade (RQ), contendo padrões de referência para a avaliação dos cursos lato sensu em Saúde Pública e/ou Coletiva; e o 'Manual da Acreditação Pedagógica', contendo as formas de verificação, instrumentos que foram produzidos pelo GT de Acreditação Pedagógica e referendados pelas instâncias coordenadoras desse processo. Sobre a utilização desses documentos, as evidências mostraram somente pontos positivos acerca deles. Entretanto, há menções à ausência de espaço público que assegure transparência ao processo de acreditação. A ausência de uma plataforma eletrônica foi mencionada como um fator limitante. Esses resultados são reiterados com os resultados de Tavares \& $\operatorname{Sin}^{\mathbf{1 0}}$, que evidenciam a transparência no processo de acreditação pedagógica em avaliação realizadas na Europa, pois, desde o momento de adesão, é possível para os atores o acompanhamento de todo o processo via plataforma eletrônica.

O processo é então concluído com a avaliação externa, após a análise dos relatórios de auto avaliação e dos documentos enviados pela
Escola, como forma de conferir a validade a ele. Os avaliadores externos produzem um relatório, contendo os resultados da visita, e fazem recomendações para subsidiar a análise do Comitê de Experts, no momento da decisão final.

\section{Acreditação pedagógica: experiência do processo de acreditação e proble- mas encontrados}

$\mathrm{O}$ processo de acreditação desenvolvido no âmbito do CAP/Abrasco envolveu cinco cursos na área da saúde coletiva, de distintas áreas de concentração, quais sejam: Vigilância em Saúde (Escola de Saúde Pública do Ceará ESP-Ceará), Tecnologias de Saneamento e Promoção da saúde (Ensp-Rio), Saúde Pública propriamente dita e Gestão do SUS e suas equipes gestoras. Em todos os casos, o processo avaliativo foi norteado pelo RQ, consideradas as três dimensões de análise: pedagógica, infraestrutura e gerencial, que se desdobram em 55 sub-dimensões.

Dadas as diferenças entre as escolas e cursos envolvidos no processo de acreditação, essas instituições expressam, em diferentes proporções, os problemas relativos às três dimensões avaliadas, considerando o caminho da qualidade a ser trilhado. No que tange ao Projeto Político Pedagógico (PPP) das Escolas, alguns cursos avaliados apresentaram dissonância com as propostas institucionais mais gerais das Escolas sob acreditação, sendo esse problema recorrente entre os cursos avaliados. Além disso, o PPP representa um instrumento não conhecido por todos os envolvidos no curso.

A análise do material produzido pelos avaliadores externos revela que, na dimensão pedagógica, todos os cursos apresentam certos limites, mas também apresentam número expressivo de fortalezas que expressam inovações tecnológicas nos processos de ensino, por dentro dos cursos. Há destaques quanto às metodologias utilizadas, à estrutura dos cursos, à qualidade do corpo docente, entre outros pontos fortes.

Destaca-se, entre os problemas de ordem pedagógica, a falta de correlação da missão 
institucional em relação ao PPP, assim como o seu desconhecimento pelas equipes envolvidas na organização dos cursos. No que concerne à missão institucional, o RQ é explicito, sendo esse elemento considerado crítico pelos documentos analisados. Muito embora os problemas tenham sido explicitados nesses dois pontos, a dimensão pedagógica aparece como um ponto forte em todos os relatórios analisados.

A adoção de mecanismos de avaliação aparece como um elemento deficiente em todas as dimensões avaliadas. Apresenta-se como uma lacuna, em todas os relatórios de avaliação externa, aparecendo como ausência de avaliação de desempenho institucional, de avaliação do curso em sua dimensão mais ampla, acompanhamento de egressos e ausência de instrumentos avaliativos básicos. A ausência de processos avaliativos aparece como uma lacuna importante em várias dimensões no SUS $^{12}$, bem como nos processos educativos ${ }^{13}$.
A dimensão gerencial tem como ponto mais crítico o desconhecimento da missão institucional pelos atores institucionais que desenvolvem o curso, indicando a necessidade de adotar estratégias integradoras entre os gestores dessas instituições e equipes docentes, estudantes e ex-alunos.

Na sequência, a análise de desempenho, ausente em quase todas as instituições que tiveram curso avaliado, aparece como problema recorrente. Ademais, a gestão do curso aparece como não participativa em várias das experiências analisadas, contrariando a tradição da área de formação em saúde pública no Brasil e os princípios do próprio SUS.

$\mathrm{Na}$ infraestrutura, os problemas relativos à sala de aula, estrutura dos banheiros, biblioteca e outros são diferentes entre si e se relacionam com a heterogeneidade das instituições avaliadas.

Quadro 1. Problemas relativos à acreditação pedagógica no Brasil

\begin{tabular}{ll}
\hline Dimensão & Problemas encontrados \\
\hline Pedagógica & Deficiência nos PPP de aportes teóricos da educação \\
& Deficiência dos instrumentos de avaliação no que tange à coordenação e à gestão do curso \\
& Pouca apropriação do corpo docente do projeto pedagógico do curso \\
Gerencial & Desconhecimento da missão institucional por atores do curso \\
& Incipiente desenvolvimento de mecanismos e instrumentos de avaliação \\
& Falta de regularidade nos processos de avaliação \\
& Avaliação focada no plano individual e não institucional \\
& Inexistência de avaliações voltadas para os egressos \\
& Falta de discentes nos espaços decisórios dos cursos \\
& Baixa capacidade de articulação interinstitucional \\
& Espaço deficiente da biblioteca \\
& Espaço deficiente de convivência \\
& Incipiente sistema de avaliação acadêmico \\
& Deficiência das instalações prediais \\
& Número de trabalhadores efetivos insuficiente \\
& Número de docentes em processo de aposentadoria \\
& Tímida utilização de tecnologias digitais \\
& Incipiente desenvolvimento da área de Tecnologia da Informação (TI)
\end{tabular}

Fonte: Elaboração própria baseada em relatórios de avaliação externa. 


\section{Efeitos da acreditação pedagógica no Brasil}

Pode-se afirmar que a implantação da acreditação pedagógica, no período referido neste artigo, apresentou efeitos positivos para as instituições e para os atores envolvidos. Embora tenham sido identificados distintos problemas, o processo vivenciado foi capaz de mobilizar os atores a construir efeitos positivos para as escolas, para os trabalhadores e para o SUS.

Para as escolas que passaram pelo processo, a autoavaliação representa um momento estratégico de encontro dos atores institucionais; momento de autoconhecimento em que, por meio da produção do relatório de autoavaliação, identificam-se as fraquezas e as fortalezas contidas no curso, na equipe e na instituição. Para os atores institucionais, a autoavaliação representa a 'alma' da acreditação pedagógica.

Os resultados produzidos no que concerne à autoavaliação, por este trabalho, são reiterados pela pesquisa de Hortale, Obaddi \& Ramos ${ }^{5}$. As autoras consideram que a Acreditação é um método de estímulo, avaliação e certificação da qualidade e caracteriza-se como um processo educacional que introduz nas instituições a cultura da qualidade.

Para as Escolas e Centros Formadores de Saúde Pública que constituem a RedEscola, as evidências apontam também que houve o envolvimento de todos os atores da instituição, enredados ou não com o curso no processo de avaliação, no momento da produção do relatório de autoavaliação. Esse momento representou um trabalho coletivo de negociação daqueles envolvidos com o curso, com a emergência de vários problemas do cotidiano e que encontraram nesse momento uma oportunidade de explicitação. A análise dos documentos revelou, ainda, que a produção do relatório de autoavaliação no processo de Acreditação define pontos estratégicos para elaboração de propostas que contribuirão para os avanços no caminho da qualidade. Segundo Mendes e cols..$^{14}$, a autoavaliação corresponde a uma estratégia participativa e na qualidade negociada como tendência das políticas de avaliação institucional. Representa também uma forma de resistência à avaliação ranqueadora, muito comum nos sistemas avaliativos.

No que tange ao arcabouço teórico e metodológico que embasa a acreditação pedagógica, esse processo é considerado apropriado para os distintos contextos e disparidades regionais existentes no Brasil. Como consequência, a acreditação pedagógica possibilita que cada escola, por meio dos seus cursos, após ser submetida à Acreditação, possa adotar diferentes estratégias para melhoria contínua da qualidade, a partir da realidade local, constituindo assim processos singulares. Trata-se, portanto, da incorporação da acreditação pedagógica como estratégia da melhoria contínua da qualidade, no âmbito dos cursos e das instituições avaliadas.

Mendes \& cols. ${ }^{14}$ destacam que, em geral, as políticas avaliativas legitimam as avaliações centradas nos produtos, portando credibilidade aos testes padronizados, proliferando uma 'avaliocracia'. Apresentam, entretanto, uma possibilidade ao entender a avaliação como uma concepção de 'Qualidade Negociada' que, em conjunto com os diferentes atores da instituição, produz novas relações dentro e fora das escolas, reforçando a aprendizagem estratégica e a competência coletiva a favor da instituição.

Cabe considerar também que, nesse processo, houve ganhos relativos aos trabalhadores das escolas do SUS. Para eles, identificam-se as reflexões sobre os processos de trabalho, por meio do curso e do reconhecimento das fragilidades pessoais em relação ao processo institucional. Trata-se, então, de uma atividade de educação permanente, ao tomar o processo de trabalho como elemento de reflexão para a ação, nesse caso, seus resultados produzindo formulações de melhorias que propõem a organização de aspectos fundamentais à dinâmica dos cursos e, fundamentalmente, à vida das Escolas. Além disso, destaca-se o fenômeno da produção coletiva de consensos, que ocorre por meio dos procedimentos de avaliação para a acreditação.

A análise do material empírico revelou que há também efeitos positivos para o SUS. 
Com relação ao modelo de atenção à saúde, a qualificação dos processos de trabalho e dos processos educativos incidem sobre as práticas de saúde desenvolvidas pelos trabalhadores no sistema de saúde. Há que se destacar nesse caso, também, a seleção de prioridades de avaliação, articulando as necessidades de formação e os cursos de especialização desenvolvidos, uma vez que a qualificação dos trabalhadores resulta em perfis mais adequados aos princípios e diretrizes do SUS. Aumentam, por sua vez, por meio da qualificação, a capacidade de governo dos trabalhadores do âmbito das escolas.

De modo geral, a reflexão e a implantação de estratégias para a melhoria contínua da qualidade dos processos educativos e das instituições formadoras não universitárias resultam na melhoria do SUS. Em última análise, o estabelecimento de um processo avaliativo, não ranqueador como a acreditação pedagógica e de valorização dos diferentes contextos e situações locais, permite o fortalecimento das instituições e da RedEscola.

Para o GT Trabalho e Educação da Abrasco, a Acreditação foi priorizada como objeto de análise, propiciando a instituição de uma linha de atuação que está articulada à área de avaliação em educação do GT. Além disso, os debates construídos entre o GT e as política em curso na esfera do trabalho e da educação na saúde mostraram interfaces entre a acreditação pedagógica e a Política de Educação Permanente, operadas pelas instituições das secretarias estaduais e municipais de saúde, estabelecendo elos importantes entre instituições formadoras do SUS e associações atuantes na formulação das políticas de saúde no âmbito da sociedade civil, como a Abrasco.

\section{Considerações finais}

Embora sejam reconhecidos os entraves iniciais estabelecidos para a implantação da acreditação pedagógica no Brasil, com a acomodação das culturas institucionais dos atores envolvidos na governança e com as necessidades que foram indicando os caminhos dos inúmeros ajustes efetuados, considera-se o processo exitoso.

No período de 2014 a 2018, após quatro anos de implantação, houve acreditação de cinco cursos das Escolas de Saúde Públicas do SUS; foram mobilizadas 14 escolas para a Acreditação; construiu-se um banco de avaliadores externos e criou-se um conjunto de instrumentos norteadores para a acreditação pedagógica, como o RQ e o 'Manual de Acreditação'.

Vale ressaltar ainda a organização de uma estrutura de governança composta por um Conselho de Acreditação, um Comitê de Experts e uma Coordenação Executiva, além de um grupo de avaliadores externos formados para a tarefa de avaliação externa; e outros formados e experienciados ou com vivência na atividade são pontos positivos. Isso possibilitou a geração de um acúmulo técnico e científico nos sujeitos que desenvolvem a acreditação no Brasil.

Entre os desafios para os próximos anos, coloca-se como imperioso construir a sustentabilidade e independência financeira do processo e da Agência, tendo em vista o estabelecimento de um SNA, levando em consideração as suas bases teóricas e conceituais, notadamente a transparência, credibilidade, ética e confiabilidade pública. Para tanto, há que se avançar na inserção de novos objetos para acreditação, como graduações, escolas e residências, entre outros. Ademais, o estabelecimento de critérios de monitoramento e avaliação dos planos de melhoria da qualidade elaborados pelas instituições que passaram por acreditação pode ser uma estratégia de melhoria da qualidade no SUS, além da aproximação entre os processos de acreditação e a PNEPS.

Há ainda que se considerar a necessidade de informatizar o processo, para reduzir o longo tempo de acreditação. Desse modo, outro elemento a ser valorizado, nos próximos passos, é a constituição de uma plataforma eletrônica para acelerar e dar transparência aos processos de acreditação e um esforço no sentido de ampliar a formação de avaliadores. 
No processo de implantação do SUS, nos últimos 30 anos, muitos investimentos foram feitos na organização de Escolas Estaduais e Municipais de Saúde Pública ou afins, no fortalecimento das estruturas universitárias e de ensino técnico que apoiam o sistema e na diversificação de programas de formação, consoantes com as políticas públicas delineadas nesse percurso. A consolidação dessas estruturas essenciais à vitalidade do SUS passa pela incorporação da cultura avaliativa como fundamental; e o processo de acreditação pedagógica se apresenta como um exemplo virtuoso a ser explorado.

\section{Colaboradores}

Matos C (0000-0002-8131-4831)*, Pinto ICM (0000-0002-1636-2909)* e Nunes TCM (0000-0001-5631-5085)* contribuíram igualmente na elaboração do manuscrito.

\section{Referências}

1. Paim JS. Sistema Único de Saúde (SUS) aos 30 anos. Ciênc. Saúde Colet. 2018; 23(6):1723-28.

2. Santos NR. SUS 30 anos. O início, a caminhada e o rumo. Ciênc. Saúde Colet. 2018; 23(6):1729-36.

3. Nunes TCM, Fagundes TLQ, Soares CL. Saúde Coletiva: A Abrasco em 35 anos de História. Rio de Janeiro: Fiocruz; 2015. p.151-67.

4. Ceccim RB. Educação permanente em saúde: desafio ambicioso e necessário. Interface Comunic. Saúde, Educ. 2005; 9(16):161-168.

5. Hortale VA, Obbadi M, Ramos CL. A acreditação e sua implementação na área de ensino pós-graduado em Saúde Pública. Cad. Saúde Pública. 2002; 18(6):1789-1794.

6. Alkhenizan A, Shaw C. Impact of accreditation on the quality of healthcare services: a systematic review of the literature. Ann Saudi Med. 2011; 31(4):407-416.
7. Braithwaite J, Greenfield D, Westbrook J, et al. Health service accreditation as a predictor of clinical and organisational performance: a blinded, random, stratified study. Qual Saf Health Care. 2010; 9:14-21.

8. Matus C. Política, planejamento e governo. 2. ed. Brasília, DF: Instituto de Pesquisa Econômica Aplicada; 1996.

9. Teixeira CF, organizadora. Planejamento em Saúde: conceitos, métodos e experiências Salvador: EDUFBA; 2010.

10. Cardoso S, Rosa MJ. European Policy Implementations: challenges for higher education Quality Assurance. In: Sin C, Tavares O, Cardoso S, et al., editors. European higher education and the internal market: tensions between European Policy and National Sovereignt. Switzerland; Springer Nature; 2018. p. 337-360.

11. Tavares O, Sin C. The visible hand of the Market 
in european higher education policies. In: Sin C, Tavares O, Cardoso S, et al., editors. European higher education and the internal market: tensions between European Policy and National Sovereignt. Switzerland; Springer Nature; 2018. p. 363-368.

12. Reis AT, Amaral MA, Oliveira PTR. IDSUS: Índice de Desempenho do Sistema Único de Saúde. Brasília, DF: Ministério da Saúde; 2012.
13. Cavalheiro MTP, Guimarães AL. Formação para o SUS e os desafios da integração ensino-serviço. Caderno FNEPAS. 2011; 1:19-27.

14. Mendes GSCV, Caramelo J, Arelaro LRG, et al. Autoavaliação como estratégia de resistência à avaliação externa ranqueadora. Educação e Pesquisa. 2015; 41(spe);1283-1298.

Recebido em 19/12/2018

Aprovado em 18/04/2019

Conflito de interesses: inexistente

Suporte financeiro: não houve 